\title{
Componentes psicosociales y su influencia en el rendimiento académico de los estudiantes de la Unidad Educativa Santa Rosa, Tungurahua Ecuador.
}

Componentes psicosociales y su influencia en el rendimiento académico de los estudiantes de la Unidad Educativa Santa Rosa, Tungurahua Ecuador.

Raynier Montoro Bombú ${ }^{1}$, Byron Enrique Mora Aviléz ${ }^{2}$, Lincoln José Adolfo Torres Valencia $^{3}$, Luis Ricardo Villacis Monar ${ }^{4}$

\begin{abstract}
. DOI: https://doi.org/10.33262/cienciadigital.v2i4.306

The objective of this study was to analyze the existing relationships between the psychosocial components and their influence on the academic performance of the students of the Santa Rosa Educational Unit. To do this, academic performance is declared as a dependent variable while the independent variable was directly related to the indicators that represent the pedagogical, psychosocial and sociodemographic dimensions that were measured in the student surveys. A survey was applied to the students of the higher basic level of the Santa Rosa Educational Unit of the Ambato canton of Tungurahua province. The sample consisted of 213 of the different parallels in ages between 13 and 15 years of age. Among the main methods used are analysis and synthesis, induction deduction, analysis of documentary sources and the survey. The selection of the sample was intentionally stratified by complete conglomerates, taking the parallels as conglomerates and the regions of coexistence whether rural or urban as strata. Seeking representation of different sectors of the population. The surveys were designed taking into account three dimensions of interest for the Educational Unit: pedagogical, psychosocial and sociodemographic factors. To carry out the statistical analysis of the dependent variable, multilevel regression models were applied. This

\footnotetext{
${ }^{1}$ Universidad Técnica De Ambato. Carrera de Cultura Física y Pedagogía de la Actividad Física, Ambato, Ecuador.r.montoro@uta.ed.ec

${ }^{2}$ Universidad Técnica De Ambato. Carrera de Cultura Física y Pedagogía de la Actividad Física, Ambato, Ecuador. byrforce1@ hotmail.com

${ }^{3}$ Universidad estatal de Guaranda. Facultad de Ciencias de la Educación, Guaranda, Ecuador lincoln.torresvalencia@hotmail.com

${ }^{4}$ Universidad estatal de Guaranda. Facultad de Contabilidad Auditoria y Administración, Guaranda, Ecuador rvillacism@hotmail.com
} 
model allowed us to establish the relationship between the dependent and independent variable where the main results are obtained. The value of the variance explained for this regression model is accepted, since there is a $71.3 \%$ relationship between the psychosocial components that can be explained by the set of independent variables included in the model. It is obtained as main conclusions Better performances in students were given in those who lived in urban communities and had no work commitments after the academic days.

Keywords: Academic performance, psychosocial factors

\section{Resumen.}

El objetivo de este estudio fue analiza las relaciones existentes entre los componentes psicosociales y su influencia en el rendimiento académico de los estudiantes de la Unidad Educativa Santa Rosa. Para ello se declaran como variable dependiente el rendimiento académico mientras la variable independiente estuvo directamente relacionada con los indicadores que representan las dimensiones pedagógicas, psicosociales y sociodemográficas que fueron medidas en las encuestas a los estudiantes. Se aplicó encuesta a los estudiantes del nivel básico superior de la unidad Educativa Santa Rosa del cantón Ambato de la provincia de Tungurahua. La muestra estuvo constituida por 213 de los diferentes paralelos en edades entre los 13 y los 15 años de edad. Dentro de los principales métodos utilizados se encuentran el análisis y síntesis, la inducción deducción, análisis de fuentes documentales y la encuesta. La selección de la muestra fue de forma intencional estratificada de conglomerados completos, tomando los paralelos como conglomerados y las regiones de convivencias bien sean rural o urbano como estratos. Buscando así representación de los diferentes sectores de la población. Las encuestas fueron diseñadas tomando en cuenta tres dimensiones de interés para la Unidad Educativa: factores pedagógicos, psicosociales y sociodemográficos. Para llevar a cabo el análisis estadístico de la variable dependiente se aplicaron modelos de regresión multinivel. Este modelo nos permitió establecer la relación entre la variable dependiente e independiente donde se obtiene como principales resultados El valor de la variancia explicada para este modelo de regresión es aceptada, puesto que existe un $71,3 \%$ de relación entre los componentes psicosociales que puede explicarse por el conjunto de variables independientes incluidas en el modelo. Se obtiene como principales conclusiones Mejores rendimientos en estudiantes de estaban dados en aquellos que vivían en las comunidades urbanas y que no tenían compromisos laborales luego de las jornadas académicas.

Palabras Claves: Rendimiento académico, Factores psicosociales. 


\section{ISSN: 2602-8085}

Vol. 2, N4, p. 281-291, octubre -diciembre, 2018

Introducción.

Conclusiones.

Referencias Bibliográficas. 


\section{Para citar el artículo indexado.}

Montoro R., Mora B., Torres L. \& Villacis L., (2018) Componentes psicosociales y su influencia en el rendimiento académico de los estudiantes de la Unidad Educativa Santa Rosa, Tungurahua Ecuador. Revista electrónica Ciencia Digital 2(3), 281-291. Recuperado desde:

http://www.cienciadigital.org/revistascienciadigital/index.php/CienciaDigital/article/view/7 $0 / 65$

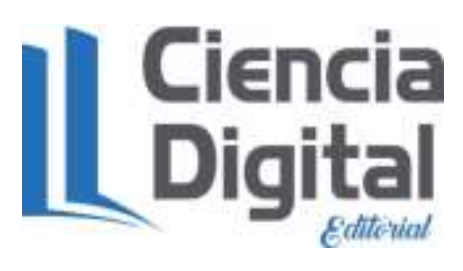

El artículo que se publica es de exclusiva responsabilidad de los autores y no necesariamente reflejan el pensamiento de la Revista Ciencia Digital.

El articulo queda en propiedad de la revista y, por tanto, su publicación parcial y/o total en otro medio tiene que ser autorizado por el director de la Revista Ciencia Digital.
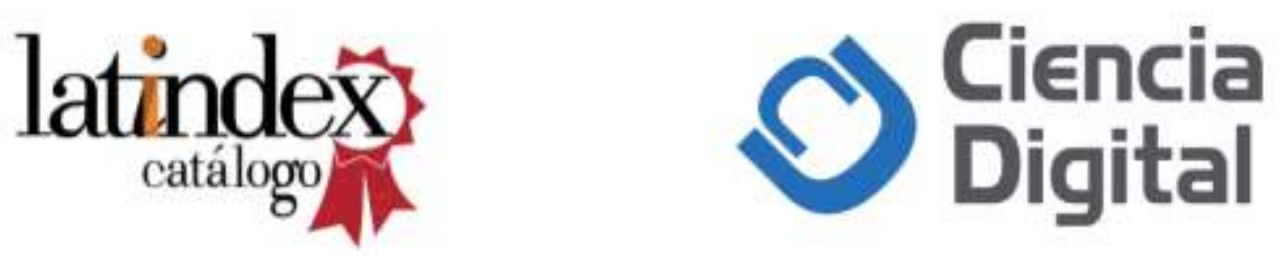